\title{
Problèmes actuels en mécanique dans le domaine ferroviaire : vers l'omniprésence du non-linéaire et du stochastique
}

\author{
Pierre-Etienne Gautier ${ }^{a}$ \\ SNCF, Direction de l'Innovation et de la Recherche, 45 rue de Londres, 75379 Paris Cedex 8, France
}

Reçu le 7 juillet 2010, accepté le 9 juillet 2010

\begin{abstract}
Résumé - Les problèmes actuels en mécanique dans le domaine ferroviaire font intervenir de manière intensive des non-linéarités, que ce soit dans le comportement des composants du système (ex. ballast...), ou aux interfaces. Par ailleurs, la prise en compte de l'aléatoire dans la modélisation devient incontournable, tant pour les sollicitations du système que pour la variabilité de ses caractéristiques. Ces deux tendances sont illustrées sur des exemples de problématiques actuelles : dynamique des véhicules et de l'infrastructure, crissement. .
\end{abstract}

Mots clés : Non-linéarités / stochastique / granulaire / recalage / dynamique ferroviaire / crissement

\begin{abstract}
Open questions in mechanics applied to railways: towards a prominence of nonlinear and stochastic approaches. Current open questions in mechanics in the railway domain intensively involve non-linear approaches either in the description of the behaviour of the system components (e.g. ballast...) or in the interfaces. Besides, stochastic approaches for modelling cannot be avoided, either for taking into account system excitation, or for modelling its intrinsic variability. Those two main drivers for current developments will be illustrated on current developments: vehicle and infrastructure dynamics, brake screech, ...
\end{abstract}

Key words: Nonlinearity / stochastic / discrete element modelling / model updating / rail vehicle dynamics / infrastructure dynamics / brake screech / cross wind

\section{Introduction}

Les problèmes actuels de mécanique dans le domaine ferroviaire sont actuellement sous-tendus par les enjeux majeurs suivants :

- augmenter la disponibilité et réduire significativement des coûts de maintenance, tant du matériel roulant que de l'infrastructure, tout en maintenant la sécurité dans des conditions de fonctionnement de plus en plus exigeantes, voire vis-à-vis de l'occurrence d'évènements extrêmes (tempêtes...). Ceci implique de mieux connaître quantitativement le comportement à la fatigue de ces deux systèmes, et leur interaction, dans des conditions de plus en plus sévères;

- gagner en performances, que ce soit en capacité en termes de nombre de voyageurs transportés pour les trains régionaux, ou en vitesse, en maintenant, voire en augmentant la capacité pour les TGV, et réduire

\footnotetext{
a Auteur pour correspondance :

pierre-etienne.gautier@sncf .fr
}

significativement le temps de développement et d'homologation des trains;

- gagner en performances pour l'infrastructure, dans des conditions de charge plus sévères et plus intenses.

Les problématiques scientifiques que ces enjeux soulèvent font apparaître, entre autres, le besoin de mieux connaître et simuler numériquement le comportement du système véhicule-voie, notamment ceux des sous-systèmes (frein à disque, voie ballastée...) ou interfaces critiques (contact roue-rail, pantographe-caténaire). Ces progrès nécessaires dans les connaissances et la simulation portent essentiellement actuellement sur les deux champs suivants :

- les phénomènes non linéaires qui interviennent soit à l'interface, soit dans le comportement des sousensembles : il est nécessaire de mieux cerner expérimentalement et de représenter avec le maximum d'efficacité (et à coût numérique minimum dans les modèles) ces comportements non linéaires tant à l'échelle locale (contact roue-rail, frottement entre grains de ballast) qu'à une échelle plus globale (véhicule et voie). Plus généralement, il s'agit 


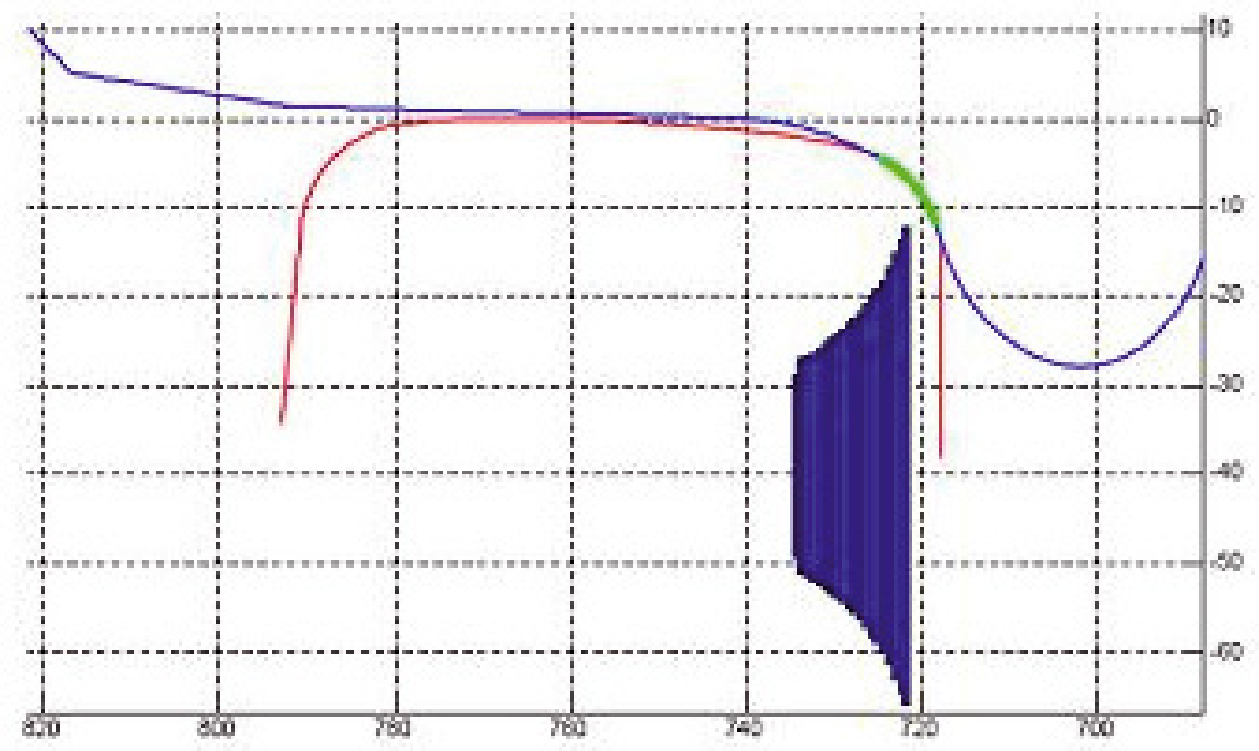

Fig. 1. Visualisation des profils en coupe de la roue et du rail et de l'empreinte du contact roue-rail dans le cas d'un déplacement latéral de la roue amenant la zone de contact sur les flancs de la roue et du rail (zone verte) : la zone de contact est très différente de l'ellipse de Hertz.

de gérer simultanément les analyses, tant à l'échelle locale qu'à l'échelle globale. C'est cette dualité des approches dans une approche complète qui permet d'appréhender de manière correcte la prévision du comportement dans le temps des sous systèmes (tassement de la voie, fatigue des roues et des rails) et donc permettra d'améliorer tant la conception que la maintenance des véhicules et de la voie;

- la prise en compte des effets aléatoires, soit dans les lois de comportement des sous-ensembles (variabilité des sols constituant l'infrastructure...) soit dans les sollicitations des systèmes (défauts de voie, vents latéraux extrêmes...),

La prise en compte de ces deux axes de progrès sera illustrée dans ce qui suit à travers des exemples portant sur les questions de comportement dynamique des véhicules ferroviaires et de crissement des freins à disques d'une part, puis de sujets liés à la voie ballastée et à la sécurité des circulations vis-à-vis des vents latéraux d'autre part.

\section{Modélisation du comportement dynamique des véhicules du point de vue sécurité de marche}

\subsection{Problématique}

La connaissance du comportement dynamique des véhicules (forces d'interaction entre le véhicule et la voie, accélérations sur différents éléments et en particulier la caisse) est importante, non seulement pour des raisons de confort mais aussi de sécurité. Ce problème se traduit au niveau de la modélisation par un problème de dynamique multicorps, comprenant un certain nombre de non linéarités. Ces dernières, d'un point de vue pratique, se situent majoritairement au niveau du contact roue-rail, et dans les caractéristiques de certaines liaisons.

$\mathrm{Au}$ niveau du contact roue-rail, les non linéarités ont été depuis longtemps prises en compte dans les codes de simulation : le point de contact se déplaçant le long des profils de la roue et du rail, il est important de prendre en compte les profils réels des roues et des rails, ce qui était fait classiquement pour les roues, mais a nécessité récemment le développement de moyens de mesure au défilé avancés pour ce qui concerne le rail. Ensuite, la zone de contact roue-rail peut être de forme et dimensions variables, en tout cas différente de l'ellipse de Hertz classique (Fig. 1) : le calcul approché de référence de la zone de contact et de la force d'interaction associée (algorithme CONTACT, ou sa version simplifiée FASTSIM développés par Kalker [1]) étant trop lourds à mettre en œuvre pour la majorité des situations de simulations industrielles, un algorithme («STRIPES ») permettant le même calcul en subdivisant la zone de contact en bandes a été récemment développé [2].

Ensuite, la loi de contact présentant une saturation pour les vitesses de glissement relatif roue-rail importantes (Fig. 2), des formulations numériques ou semi empiriques (par ex. Vermeulen et Johnson [3]) ont été développées.

Enfin, les non linéarités résident aussi dans l'existence de butées pour limiter les déplacements latéraux relatifs entre la caisse et le bogie par ex. ou dans les caractéristiques de certains composants (amortisseurs « anti-lacet » placés entre caisse et bogie destinés à retarder l'apparition avec l'augmentation de la vitesse de 


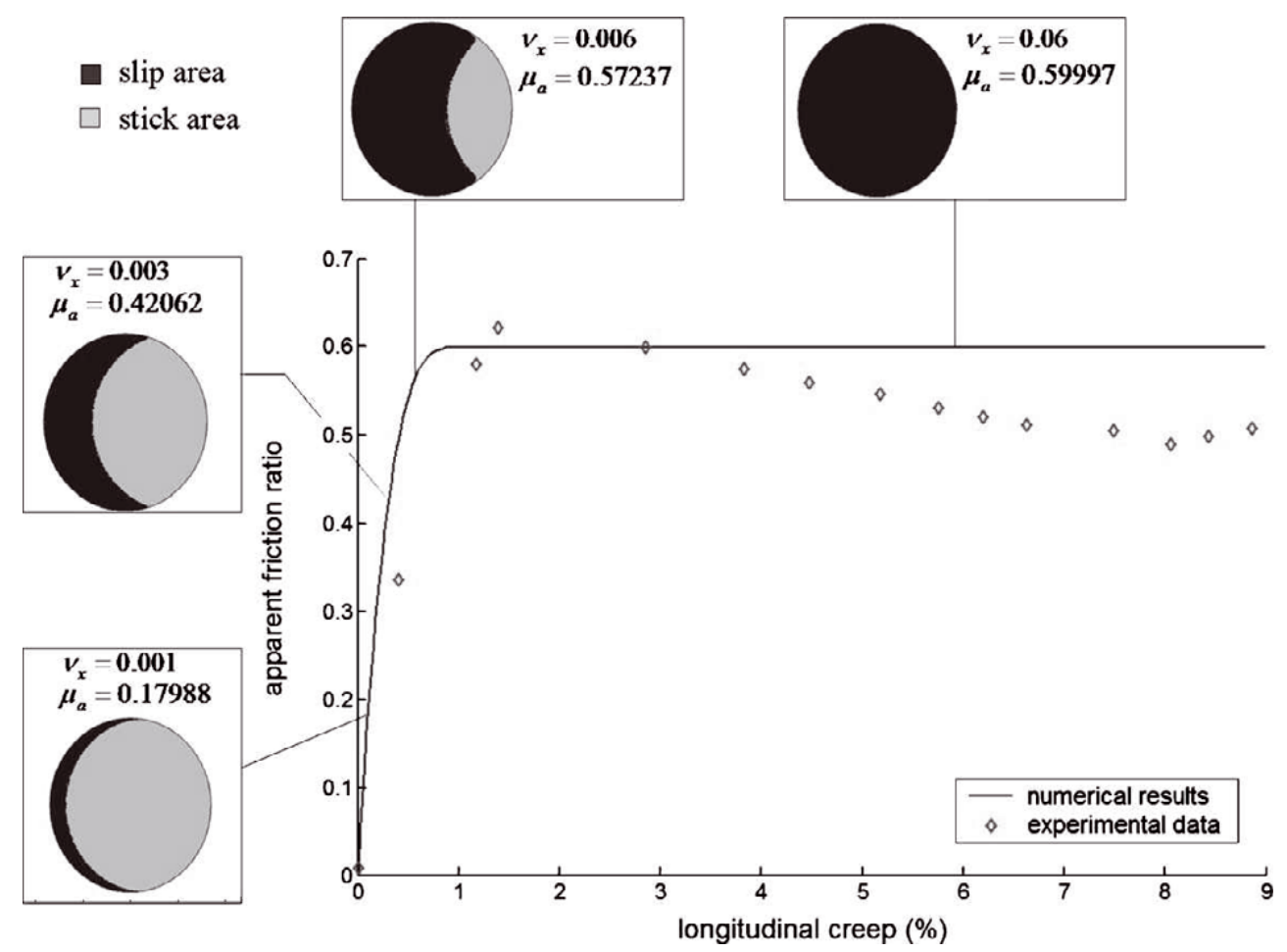

Fig. 2. Coefficient de frottement en fonction du glissement, d'après [Piotrowski, Wheel rail contact models..., VSD 43 (2005) 455-483].

circulation des cycles limites qui vont être évoqués dans ce qui suit).

Pour l'essentiel des applications qui vont être mentionnées dans la suite, stabilité de marche et confort dynamique, les fréquences mises en jeu sont inférieures à la dizaine de $\mathrm{Hz}$, ce qui fait que pour la modélisation des véhicules, une approche de type solide rigide peut suffire. Néanmoins, la prise en compte de la flexibilité des caisses de véhicules est de plus en plus nécessaire, notamment lorsque sont étudiées les vibrations de caisses de véhicules à deux niveaux entre 5 et $20-30 \mathrm{~Hz}$. Elle a de ce fait été implémentée dans un certain nombre de codes en dynamique ferroviaire.

L'ensemble des non linéarités mentionnées ci-dessus est à l'origine, autour d'une certaine vitesse, dite « vitesse critique », de cycles limites d'amplitude « importante » pour les oscillations de l'essieu dans la voie pouvant conduire à des efforts importants sur la voie tendant à la déstabiliser [4] (Fig. 3).

Selon les vitesses de circulation, les phénomènes de bifurcation à l'origine de ces cycles limites ont été étudiés, essentiellement à travers des approches numériques $[5,6]$. Des phénomènes de type chaotique ont même ainsi pu être mis en évidence sur des exemples numériques, même si, dans la pratique, les phénomènes observés sont essentiellement du type cycle limite. Le wagon à simple essieu et à suspension à ressorts à lames présente des diagrammes de bifurcation particulièrement complexes [7] dépendant des caractéristiques de la voie.

Dans la pratique, on cherche à se placer pour les vitesses de circulation commerciales à une vitesse inférieure

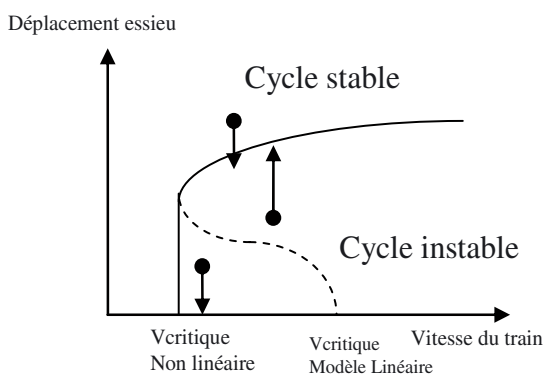

Fig. 3. Comportement non linéaire d'un essieu : le cycle limite théorique.

à la « vitesse critique » et l'homologation des véhicules, jusqu'à présent réalisée à partir d'essais de circulation en ligne, consiste à faire rouler le véhicule sur un nombre suffisant de tronçons de voie de caractéristiques de tracé diverses (voie en alignement, courbes de différents rayons) pour estimer dans ces conditions les valeurs « extrêmes » des forces que le véhicule est susceptible d'exercer sur la voie.

Les valeurs limites admissibles sont pour l'instant issues de lois phénoménologiques du comportement de l'infrastructure (rails, traverses, ballast), à partir de la mise en commun au niveau européen de la connaissance du comportement de divers véhicules au sein de l'Union Internationale des Chemins de fer (UIC), et transcrite dans un document (fiche UIC 518 [8]) qui sert de base à la norme EN 14363 qui prescrit le mode de réalisation des essais. 
La réalisation de ces essais est un processus actuellement jugé trop long et coûteux parce qu'il demande des circulations sur des kilométrages importants de voies de caractéristiques variées. De plus, certaines conditions de circulation requises (qualité de voie couplée à certains types de courbes) ne peuvent quelquefois pas être remplies en pratique. La question s'est donc posée d'utiliser les modèles numériques de modélisation multicorps des véhicules ferroviaires pour gagner en efficacité et en robustesse dans le processus d'homologation.

Le principe n'est pas de supprimer les essais mais d'en réduire le nombre et d'utiliser la simulation pour :

- recréer des conditions d'essai (qualité de voie, paramètres du contact roue-rail) standardisées, donc permettant une évaluation plus objective vis-à-vis des grandeurs limites. En effet, certains paramètres comme le coefficient de frottement au contact rouerail ne sont pas maîtrisables lors des essais. L'objectif est donc de recréer par le calcul un essai à conditions « normées » (coefficient de frottement, qualité de la voie);

- prendre en compte la variabilité de certaines caractéristiques du véhicule (masse, inertie, caractéristiques de suspensions ...) qui peuvent varier d'un véhicule de série à l'autre;

- permettre à terme l'homologation de véhicules «peu différents » d'un véhicule de référence (véhicule issu d'une même plateforme) à partir de résultats d'essai du véhicule de référence et d'extrapolations par le calcul.

Ces objectifs sont l'objet d'un projet «DYNOTRAIN » qui a été soumis au $7^{\mathrm{e}} \mathrm{PCRD}$ et qui associe à la fois des industriels constructeurs de matériel, des opérateurs et gestionnaires d'infrastructures ferroviaires, et des instituts de recherche.

Les problématiques scientifiques ouvertes par ces ambitions sont de deux natures :

- le développement de modèles harmonisés et coconstruits entre industriels constructeurs et exploitants pose en particulier la question du recalage de ces modèles sur les essais, et la détermination des étapes et paramètres de validation du modèle, indispensable au cours de la phase de conception de véhicule, pour garantir au moment de la mise sur rail du premier prototype un comportement le plus proche possible du comportement attendu;

- l'utilisation de modèles pour prévoir le comportement de véhicules présentant des variabilités dans des conditions différentes des conditions d'essai pose la question de la validité d'un modèle apte à prévoir le comportement d'un véhicule dont les caractéristiques sont différentes de celles du modèle. Ceci peut se produire soit de manière délibérée et voulue, lorsqu'il s'agira de prévoir le comportement d'une nouvelle série de véhicules proches d'une série déjà testée soit de manière non maîtrisée, lorsque l'on teste un véhicule qui présente par nature des dispersions dues à la fabrication par rapport aux valeurs nominales prévues. Dans ces cas, on s'intéressera à la réponse d'un système dont les paramètres sont aléatoires mais dont la distribution peut être estimée, l'excitation de ce système présentant elle aussi une part stochastique : les « défauts » d'irrégularité géométrique de la voie. La problématique est alors celle de la réponse (quantités de type valeurs extrêmes, valeurs RMS filtrées...) d'un système présentant un nombre réduit mais significatif de non-linéarités, dont certains paramètres sont aléatoires, et lui-même soumis à une excitation stochastique ;

- enfin, l'estimation du coefficient de frottement entre la roue et le rail, paramètre-clé qui gouverne quand il augmente les risques de déraillement ou l'apparition des cycles limites est un problème ouvert en pratique, alors que sa connaissance sera indispensable au recalage des modèles numériques sur les valeurs mesurées.

Les réflexions concernant les derniers points en étant à leur début, la section qui suit se concentrera sur la question du recalage des modèles.

\subsection{Recalage de modèles en dynamique ferroviaire}

La problématique du recalage de modèles multicorps en dynamique ferroviaire tient essentiellement aux caractéristiques suivantes :

- méconnaissance tant au niveau de la description de comportement que des lois de certaines caractéristiques (suspensions, amortisseurs) d'éléments de suspension, voire de masse ou d'inertie des véhicules;

- non-linéarités du comportement au contact roue-rail mais aussi au niveau de certains éléments de liaison (amortisseurs, butées...) ;

- nombre de points d'observations généralement limité (30 à 50 points de mesure pour une rame TGV articulée de 8 caisses) et difficulté d'accès (du moins de manière exhaustive) à certaines grandeurs (forces au contact roue-rail, forces dans des éléments de liaison non-linéaires).

Un travail de comparaison de différents modèles utilisés dans la communauté [9] avait été réalisé sur des cas de référence. Ce travail a été poursuivi dans le cadre de l'évaluation du comportement d'un nombre plus restreint de modèles mais dans des configurations opérationnelles [10]. Les résultats montrent que les différents modèles utilisés dans la communauté se comportent de manière similaire, pour autant bien entendu que les ingrédients physiques essentiels du comportement y soient incorporés.

Plus récemment, un travail d'identification paramétrique utilisant une méthode d'état adjoint a été présenté dans le cadre de l'application à un bogie. L'extension de ce type de méthode au cas du véhicule complet est en cours. 


\section{Compréhension du crissement des freins à disques dans le contexte ferroviaire}

Le problème du crissement des freins à disques des véhicules ferroviaires, et en particulier celui des TGV, a fait récemment l'objet de travaux qui, dans un premier temps, ont visé à la compréhension de la génération du phénomène, avec pour objectif d'en tirer des pistes pour la suppression du crissement [11].

Le problème du crissement des disques de frein avait déjà été abordé pour les secteurs aérien et automobile. Par rapport à ce dernier secteur, le phénomène, dans le cas des disques TGV, se caractérise par un nombre de modes de disques plus important, exacerbé par les modes de plots de garnitures (nombre important de modes possibles associés à un nombre important de structures de petite taille). La nature des modes est aussi différente : dans l'aérien le disque vibre autour de son axe ( whirl vibration $»)$ comme un solide rigide articulé, alors que dans les cas automobile et ferroviaire, les modes impliquent la déformation du disque.

Les fréquences concernées sont différentes, les fréquences émergentes pour le cas ferroviaire se situant dans la bande [5000-15000 Hz].

Une première modélisation stationnaire, consistant à calculer les modes correspondant à la solution de frottement avec glissement stationnaire du disque entre ses garnitures à partir d'une formulation eulérienne originale du problème, mettait en évidence un nombre de modes instables largement supérieur aux modes « crissants » observés dans la pratique.

Une modélisation transitoire du phénomène a ensuite été entreprise, en s'appuyant sur des méthodes développées par M. Jean et J.J. Moreau pour la mécanique non régulière, qui permet de traiter des évènements fortement non-linéaires, comme le décollement suivi d'un choc et/ou la transition glissement/adhérence.

La projection de la solution numérique obtenue sur les modes instables a permis de faire apparaître, suivant différentes conditions initiales, les contributions, variables au cours du temps de ces différents modes instables. Les contributions des déformées modales des différents modes instables dans la forme spatiale du cycle limite ont par ailleurs été obtenues.

Les travaux se poursuivent pour améliorer l'efficacité et l'économie de la modélisation tout en prenant en compte les dispersions de caractéristiques et de fabrication des plots des garnitures, notamment. En particulier, des travaux de réduction de modèle sont en cours, qui devraient permettre d'établir les liens entre les niveaux vibratoires et l'architecture du système. En particulier, les outils en cours de développement devraient permettre, dans leur version industrielle, de contribuer au développement de nouveaux concepts de garniture «non crissantes » pour lesquels la phase de mise au point au banc, naguère très itérative, pourrait être considérablement réduite. Ces travaux s'appliquent à la fois aux disques frettés sur essieu et aux disques flasqués sur roue.

\section{Compréhension du comportement de l'infrastructure ferroviaire}

Rappelons que la voie ferrée est constituée de deux rails reposant par l'intermédiaire de semelles en caoutchouc sur des traverses, elles-mêmes posées sur un lit de ballast. Le ballast est lui-même constitué de fragments de roche dure, de granulométrie contrôlée. Sous le ballast se trouvent, au moins pour les voies à grande vitesse, deux couches de granulats appelées respectivement sous-couche et couche de forme.

Le ballast assure la résistance latérale de la voie, le drainage vis-à-vis des précipitations et présente l'avantage de pouvoir être brassé lors des opérations de maintenance de la voie appelées bourrage, permettant ainsi de reconstituer la qualité géométrique de la voie (absence d'affaissements localisés qui entraîneraient l'apparition de défauts géométriques d'alignement, eux-mêmes générateurs de variations d'effort au contact préjudiciables au confort, voire à la qualité de marche de train).

Le passage en exploitation à $270 \mathrm{~km} \cdot \mathrm{h}^{-1}$ puis $300 \mathrm{~km} \cdot \mathrm{h}^{-1}$ sur les lignes à grande vitesse a conduit après une quinzaine d'années à l'apparition de défauts qui ne pouvaient plus être corrigés par les opérations de bourrage usuelles. Un renouvellement complet de la voie a donc été nécessaire. Ces évènements ont appuyé la nécessité d'une compréhension plus poussée du comportement de la voie ballastée, notamment aux grandes vitesses.

\subsection{Connaissance expérimentale de comportement de la voie}

Un premier ensemble de travaux expérimentaux, menés à l'échelle $1 / 3$, avait permis de dégager des lois expérimentales de comportement du ballast $[12,13]$. Plus récemment, des essais à l'échelle 1 ont été réalisés, soit sur une cellule triaxiale - qui compte tenu de la géométrie du ballast doit être de taille suffisante (ex. cellule du NGI en Norvège, Fig. 4), soit sur une base cylindrique au LCPC. Il a paru plus pertinent, en particulier pour l'étude des questions liées à la grande vitesse, de recréer l'effet de la charge mobile à travers la combinaison d'efforts sur 3 vérins successifs simulant le passage de la charge mobile [14]. Le principe a aussi été appliqué dans des installations à l'échelle 1 construites en Espagne (Fig. 5) ou au Japon. Le caractère confiné du tronçon de voie reconstitué pour ces installations $(\sim 25 \mathrm{~m} \times 7 \mathrm{~m} \times 4 \mathrm{~m})$ crée cependant des phénomènes dynamiques qui demandent des analyses poussées des résultats obtenus sur ces installations, par comparaison avec les valeurs obtenues en voie. L'approche expérimentale en laboratoire reste incontournable, mais plutôt que l'utilisation directe de boucles d'essai en circuit fermé comme il en existe en Allemagne (Wildenrath), les réflexions pourraient se porter sur la combinaison de 


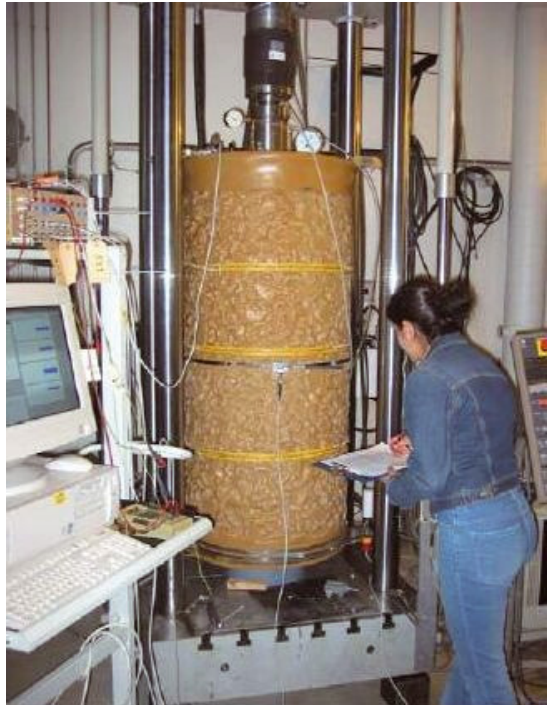

Fig. 4. Essais triaxiaux sur du ballast échelle 1 (NGINorvège).

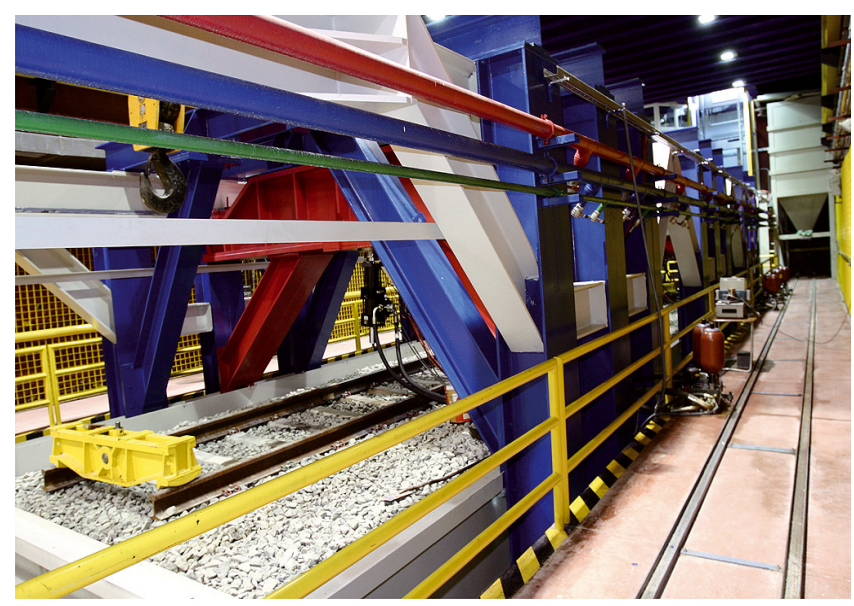

Fig. 5. Banc d'essai à l'échelle 1 de structures de voie ballastée (Cedex-Madrid).

bancs existants ou à créer testant séparément la partie plateforme et la partie «superstructure » (rails-traversesballast) de la voie ferrée.

\subsection{Modèles numériques du comportement dynamique de l'infrastructure}

En parallèle, une démarche de modélisation du comportement de l'infrastructure a été entreprise. Deux approches ont été explorées :

- une approche de type «milieux continus », plutôt orientée vers la modélisation du comportement global de l'infrastructure;

- une approche de type « milieux discrets » visant dans un premier temps à mieux comprendre le comportement du ballast à l'échelle locale.

\subsubsection{Approche « milieux continus »}

Dans le cadre de l'approche «milieux continus », un modèle de type loi de Hujeux [15] couplé à une approche éléments finis (équations intégrales de frontière) avait fait l'objet de premières investigations.

Plus récemment, des approches focalisées sur la prévision des vibrations ont été développées soit utilisant par exemple un développement de Fourier du chargement associé aux particularités de la charge mobile [16] soit tenant compte de la périodicité longitudinale de la structure de la voie [17], mais toujours dans des hypothèses de comportement linéaire pour les matériaux. Un modèle numérique utilisant des techniques de sous-structuration avait aussi été développé.

La confrontation des résultats de ces modèles avec des mesures en voie avait montré que si les grandes tendances fréquentielles en comportement sont bien captées, la comparaison quantitative des résultats des calculs aux mesures fait apparaître des différences significatives dans les amplitudes des valeurs des accélérations dans le sol [18] d'autant plus sensibles que l'on s'éloigne de l'aplomb du rail. L'origine de ces différences a été recherchée dans une non-linéarité du comportement du ballast. Un modèle élastique unilatéral («no tension ») a ainsi été développé [19].

Plus récemment, les pistes envisagées pour améliorer la pertinence des modèles ont été orientées sur la caractérisation et la prise en compte de variabilités dans le comportement du ballast, mais aussi des souscouches et couches de forme. Un développement de type «éléments finis stochastiques » est en cours de test [20]. De tels types de développements, pour être efficaces en pratique, doivent s'accompagner d'une quantification expérimentale de ces variabilités : l'utilisation de carottages répétés est longue, coûteuse et intrusive pour l'infrastructure tout en en entraînant une indisponibilité de la voie pour les circulations : une méthode originale couplant un pénétromètre dynamique à un géo-endoscope (Fig. 6) a été récemment développée, et s'est avérée pratique sur le terrain. La remontée aux caractéristiques mécaniques de base à partir des informations du pénétromètre n'est cependant pas immédiate et, en complément, l'utilisation de méthodes de type SASW pourrait être appliquée de manière plus systématique.

\subsubsection{Approche « milieux discrets »}

En parallèle, le souci de comprendre le comportement du ballast a conduit à développer des méthodes spécifiques en mécanique des milieux discrets, pour comprendre le comportement du ballast. Une première approche [21] a utilisé une méthode de type dynamique moléculaire, en représentant les grains de ballast par des polyèdres tridimensionnels. Ensuite, ayant en vue l'application de modèles de type dynamique des contacts à la modélisation de l'opération de « bourrage » du ballast, opération de maintenance visant à brasser les grains de 


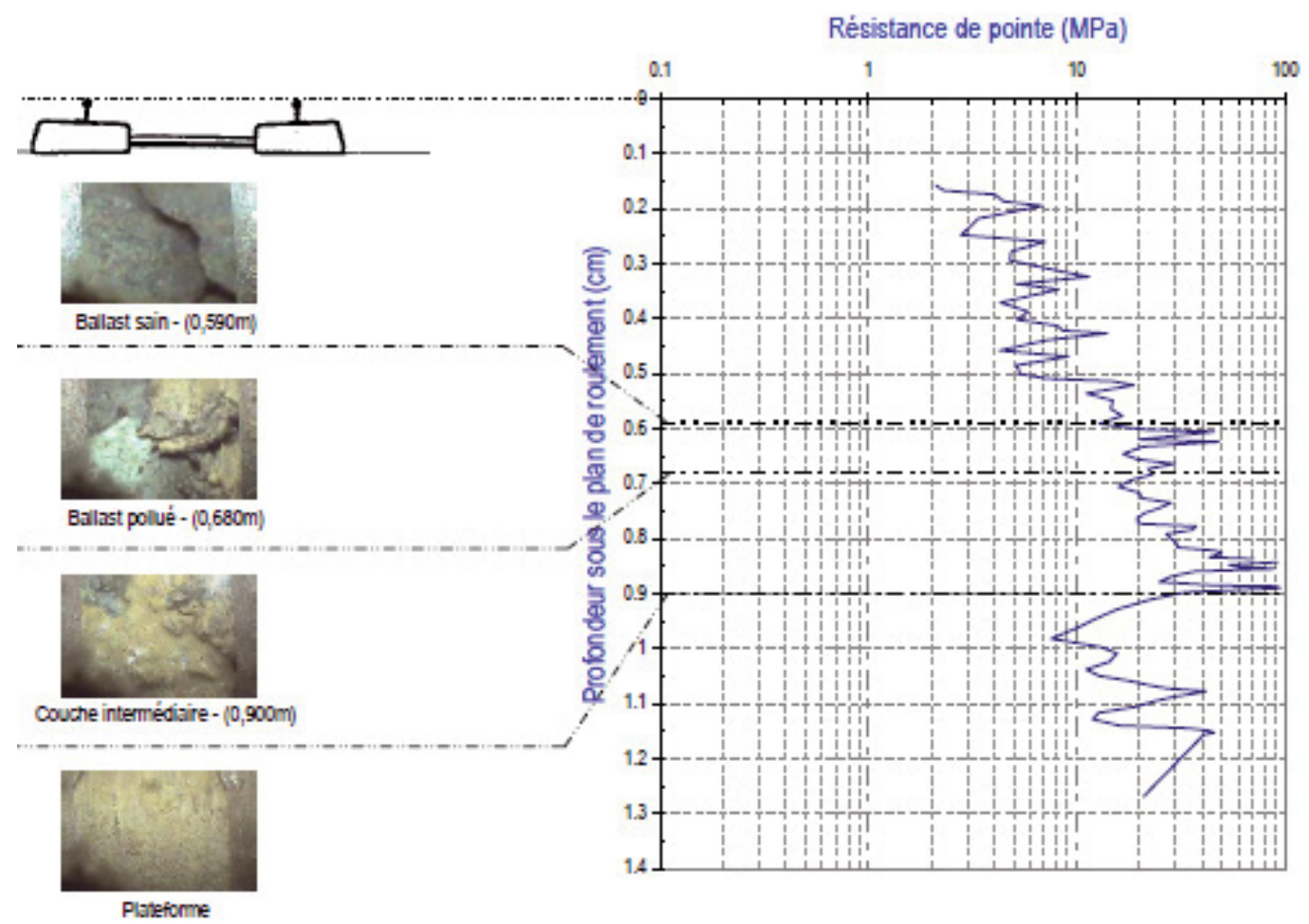

Fig. 6. Exemple de résultat obtenu par l'utilisation couplée d'un pénétromètre dynamique et d'un géo-endoscope dans l'épaisseur d'une couche de ballast.

ballast sous les traverses en ayant levé le rail et cellesci pour reconstituer la qualité géométrique de la voie, le développement d'un module de dynamique des contacts de solides tridimensionnels a été entrepris au sein de la plateforme LMGC $[22,23]$. Ce travail a permis en particulier une meilleure compréhension de la répartition des forces entre traverses et sous-couche dans l'épaisseur du ballast; il est en effet apparu qu'un petit nombre de grains inclus dans des structures dites SRL « structures rigides localisées $»$ (Fig. 7) participait effectivement à la transmission des forces [24]. Suivant la sollicitation, le type des contacts intervenant ainsi que leur orientation préférentielle a aussi été mis en évidence [25] de manière différentielle entre une géométrie sphérique et la géométrie réelle de grains de ballast.

La dynamique vibratoire de particules en milieu confiné a par ailleurs été étudiée avec ce type de méthode, en donnant des informations intéressantes pour améliorer l'efficacité de l'opération de bourrage, qui consiste à brasser le ballast sous la voie pour recréer la qualité géométrique de cette dernière [26].

Les résultats des calculs sont très dépendants du modèle local de comportement du frottement entre grains qui a été introduit. Ce modèle nécessiterait un calage expérimental plus poussé, ainsi que d'éventuels développements, en évaluant parallèlement la sensibilité des résultats globaux à ce modèle local.

Il reste aussi à faire le «pont » entre les deux approches, mieux intégrer les variabilités dans l'approche globale, et peut-être développer une approche hybride



Fig. 7. Visualisation, à partir d'une simulation par éléments discrets 3D des grains de ballast participant de manière majoritaire à la transmission des forces entre la traverse (en rouge en haut) et les couches de la plateforme.

pour intégrer le comportement du ballast dans un modèle global de voie.

\section{Sécurité des circulations vis-à-vis des vents latéraux}

La question de la sécurité des circulations à grande vitesse vis-à-vis des vents latéraux est apparue à la fin des années 1990, tant en France avec la construction de la ligne TGV Méditerranée que dans différents pays européens. Le traitement de ces problèmes, entrepris d'abord dans un cadre national, puis porté au niveau 
européen dans le cadre des Spécifications Techniques d'Interopérabilité (STI), peut être résumé en les étapes suivantes $[27-29]$ :

- la caractérisation de la sensibilité au vent des trains fait intervenir d'une part des essais en soufflerie pour connaître les coefficients aérodynamiques liées à la forme de la motrice avant, puis la simulation dynamique du déchargement lié à une rafale. Un modèle probabiliste de rafale « extrême » [30] a ainsi été introduit dans le cadre de la STI qui prescrit les caractéristiques requises pour les trains à grande vitesse européens. Une modélisation plus fine du vent (processus stochastique) a par ailleurs été utilisée dans un modèle plus fin de comportement du véhicule, conduisant à des résultats comparables [31]

- la caractérisation de l'exposition météorologique de la ligne ;

- la stratégie de protection, qui peut consister en des protections fixes permettant de réduire le risque local de déchargement, ou le risque global sur une portion de voie. Dans le cadre des lignes TGV Méditerranée et TGV Est Européen, un système de protection dynamique permet de ralentir les trains si le risque, compte tenu du vent estimé sur la ligne à partir de la mesure sur un réseau de stations anémométriques, excède une certaine valeur. Ce système est basé sur une estimation hors ligne du vent le long de la ligne, ainsi que sur la probabilité de l'évènement redouté [29].

\section{Conclusion}

Les problématiques actuelles en mécanique dans le domaine ferroviaire font intervenir de manière incontournable le non linéaire et le stochastique. Des exemples ont été présentés illustrant les tendances suivantes :

- la connaissance plus fine des non-linéarités dans le comportement aux interfaces véhicule-infrastructure est indispensable pour prévoir des phénomènes importants pour la sécurité, mais aussi pour le recalage de modèles numériques. Les cycles de conception/homologation se raccourcissant, le travail sur des modèles numériques mieux validés et tenant compte des dispersions des caractéristiques de fabrication devient incontournable, par exemple pour préparer l'homologation des véhicules;

- la connaissance du comportement de l'infrastructure (voie ballastée) nécessite de faire appel à des méthodes originales (mécanique des milieux granulaires). Plus généralement, la connaissance et la modélisation des variabilités des paramètres définissant l'infrastructure ferroviaire doivent progresser;

- tant pour la dynamique des véhicules (confort et sécurité) que pour le comportement vis-à-vis de phénomènes climatiques inhabituels (tempêtes), une approche probabiliste de la modélisation du comportement des véhicules devient indispensable.
Si les méthodes de modélisation existent, leur déclinaison aux problèmes concrets évoqués dans les exemples est en cours ou reste encore un problème ouvert. Au final leur appropriation par les praticiens du secteur reste à travailler. La prise en compte des phénomènes au niveau local (non-linéarités au contact roue-rail, frottement local entre grains de ballast) permettra, une fois intégrée au niveau global de modéliser de manière prédictive - et c'est encore un enjeu majeur - la génération de défauts dans les roues et le rail, ou la dégradation du ballast.

La connaissance des chargements réels, leur modélisation probabiliste, tout comme celle des défauts de géométrie de la voie, permettra une approche fiabiliste de la mécanique des éléments du système, permettant ainsi d'optimiser la conception, mais aussi de définir une maintenance plus appropriée, donc moins coûteuse.

\section{Références}

[1] J.J. Kalker, Three dimensional bodies in rolling contact, Kluwer, 1990

[2] X. Quost et al., Assessment of a semi-Hertzian method for determination of the wheel-rail contact patch, Vehicle System Dynamics 44 (2006) 789-814

[3] P.J. Vermeulen, K.L. Johnson, Contact of non spherical bodies transmitting tangential forces, J. Appl. Mech. 31 (1964) 338-340

[4] H. True, On the Theory of Nonlinear Dynamics and its applications, Vehicle System Dynamics 31 (1999) 393-421

[5] H. True, On the Critical Speed of High speed railway vehicles, in Noise and Vibration of high Speed Railways, R. Caçalda (ed.), Porto, 2008, pp. 149-166

[6] E. Slivsgaard, H. True, Chaos in Railway Vehicle Dynamics, in Nonlinearity and Chaos in engineering Dynamics, Thompson and Bishop (eds.), Wiley, 1994, pp. $183-192$

[7] M. Hoffmann, H. True, the dynamics of European twoaxle freight wagons with UIC Standard suspension, Vehicle System Dynamics 46 supplement (2008) 225-236

[8] UIC Code 518, Testing and approval of railway vehicles from the point of view of their dynamic behaviour-Safetytrack fatigue-Running stability, UIC Paris, 2008

[9] S. Iwnicki, The results of the Manchester benchmarks, Vehicle System Dynamics, Supplement 31 (1999) 40-48

[10] C. Funfschilling et al., Flange climb derailment simulation with two different contact models, in Proceeding WCRR 2009, Seoul, 2009

[11] X. Lorang, Instabilité vibratoire de structures en contact frottant : application au crissement des freins de TGV, Thèse de doctorat, École Polytechnique, 4 Octobre 2007

[12] N. Guérin et al., Identification expérimentale d'une loi de tassement du ballast, Revue Canadienne de Géotechnique 36 523-532

[13] V. Bodin, P. Tamagny, K. Sab, P.-E. Gautier, Fréquence critique de sollicitation lors du tassement à grand nombre de cycles d'un milieu granulaire, in Actes des journées scientifiques du LCPC, 2000, tome 2, pp. 291-296

[14] A. Al Shaer, Analyse des déformations permanentes des voies ferrées ballastées - approche dynamique, Thèse de doctorat, ENPC, Dec. 2005 
[15] J.C. Hujeux, Une loi de comportement pour le chargement cyclique des sols, Génie parasismique, Davidovici (ed.), 287, 302

[16] G. Degrande, G. Lombaert, An efficient formulation of Krylov's prediction model for train induced vibrations, J. Acoust. Soc. Am. 110 (2001) 1379-1390

[17] G. Degrande, D. Clouteau et al., A numerical model for ground borne vibrations traffic based on a periodic finite element-boundary element formulation, J. Sound Vib. 293 (2006) 645-666

[18] G. Lombaert, G. Degrande et al., The experimental validation of a numerical model for the prediction of railway induced vibrations, J. Sound Vib. 297 (2006) 512-535

[19] V.H. Nguyen, D. Duhamel, B. Nedjar, A no tension model for granular material in 2nd Europen Conference on granular Mechanics ECCM2001, June 2001

[20] N. Rhayma, Ph. Bressolette, P. Breul, M. Fogli, G. Saussine, SFE analysis of Railway Tracks, accepté à ECOSSAR, 2009

[21] X. Oviedo, K. Sab, P.-E. Gautier, Étude du comportement $\mathrm{du}$ ballast ferroviaire par un modèle micromécanique, in Actes des journées scientifiques du LCPC, 2000, tome 2, pp. 339-344

[22] G. Saussine, Contribution à la modélisation de granulats tridimensionnels : application au ballast, thèse de doctorat, Univ. Montpellier II, oct. 2004

[23] G. Saussine, F. Dubois, C. Cholet, C. Bohatier, P.-E. Gautier Modélisation du comportement du ballast par une méthode d'éléments discrets, in Actes du $6^{\mathrm{e}}$ colloque National en Calcul des Structures, PottierFerry et al. (ed.), t3, Giens, 2003, pp. 476-480

[24] G. Saussine, F. Radjai, F. Dubois, C. Cholet, P.-E. Gautier, Shear strength properties of a thin granular interface, Powder and grains, 2005

[25] E. Azema, F. Radjai, G. Saussine, Quasistatic behaviour and force transmission in packing of irregular polyhedral particles, accepted in Powder and grains, 2009

[26] E. Azema, F. Radjai, R. Peyroux, F. Dubois, G. Saussine, Vibrational dynamics of confined granular materials, Phys. Rev. E 74 (2006) 031302

[27] P.-E. Gautier et al., Protection de la ligne TGVMéditerranée basée sur une prévision temporelle des vents forts, Instrumentation Mesure Métrologie 2 (2002) $117-136$

[28] P.-E. Gautier, Fl. Sourget, E. Allain et al., Cross-wind effects on train operation, the DEUFRAKO research programme, in Proceedings WCRR 2003, EdinburghScotland, 2003

[29] F. Sourget et al., Protection de la ligne TGVMéditerranée face aux vents traversiers, in Proceedings Lambda-mu 2004, 2004

[30] W. Bierbooms et al., Modelling of extreme gusts for design calculations, New Gust final report WE01170, Delft University of Technology, 2001

[31] X. Quost, Modélisation de l'effet du vent sur les trains à grande vitesse, Étude dynamique et stochastique appliqué aux risques de renversement, Thèse de doctorat de l'École Centrale Lyon, 2005 